\title{
Outcome of living donor liver transplantation in patients of hepatic malignancy with metachronous adrenal gland metastasis: Can we achieve long term survival?
}

\author{
Ashok Thorat ${ }^{1}$, Shih-Chao Hsu ${ }^{1,2}$, Horng-Ren Yang ${ }^{1,2}$, Te-Hung Chen ${ }^{1,2}$, Kin-Shing Poon ${ }^{3}$, Ping-Chun Li ${ }^{4}$ and Long-Bin \\ Jeng ${ }^{1,2 *}$
}

${ }^{1}$ Organ transplantation centre, Taiwan

${ }^{2}$ Department of Surgery, Taiwan

${ }^{3}$ Department of Anaesthesiology, Taiwan

${ }^{4}$ Department of Cardiovascular surgery, Taiwan

*Corresponding author: Long-Bin Jeng, Organ transplantation centre, Department of Surgery, Taiwan

Submission: 海 November 19, 2018 ; Published: 海 December 18, 2018

\begin{abstract}
Hepatocellular carcinoma (HCC) is third most frequent cause of cancer-related deaths worldwide. Liver transplantation (LT) is a potentially curative treatment and is the best treatment option for patients with decompensated cirrhosis. Although advanced HCCs are considered as contraindication for LT due to dismal prognosis, certain patients of HCCs with solitary metastasis in adrenal gland without any other extra-hepatic disease can still be managed by local resection of the adrenal gland metastasis and sequential LT. Herein we present our experience of sequential 4 cases of HCC solitary metastasis to adrenal gland that were treated by local resection and LT.
\end{abstract}

Materials and Methods: Database of 937 patients that underwent LT at china medical university hospital was retrospectively analyzed. Four HCC patients that had solitary adrenal metastasis were evaluated for the outcome after living donor liver transplantation (LDLT). Three patients were diagnosed to have HCC whereas one patient was diagnosed with mixed HCC-Cholangiocarcinoma on explant pathology. All the four patients had underlying cirrhosis with solitary adrenal metastasis without any extrahepatic spread of primary disease. The adrenal gland metastasis was confirmed by pre-LT positive emission tomography (PET) scan.

Results: Four patients (mean age, 53 years; M:F, 2:2) underwent LDLT for HCC with single adrenal metastasis (right adrenal gland=2 patients; and left adrenal gland $=2$ patients) . In three patients the adrenalectomy was performed during the recipient surgery whereas one patient underwent LDLT six months after the adrenal metastasis resection (Table 1). Follow up period ranged from 9 months to 59 months. Survival in first two patients was 52 and 59 months, respectively. The first patient developed lung metastasis and expired due to overwhelming sepsis at $52^{\text {nd }}$ post-LT month. The second patient in this series continue to have recurrence free survival at 59 months post-LT. The survival in third and fourth patients was 28 months and 9 months, respectively. The fourth patient in addition had mixed HCC-cholangiocarcinoma on histopathological analysis.

Conclusion: Limited extrahepatic metastasis due to HCC can still be resected and LT can be performed if liver resection not possible with a good overall survival. The adrenalectomy can be done as a single stage procedure during LT surgery with modest long-term outcome.

Keywords: Living donor liver transplantation; Hepatocellular carcinoma; Metachronous adrenal gland metastasis; Adrenalectomy

\section{Introduction}

HCC remains a common cause of cancer related deaths in Asia [1]. Despite surveillance efforts, most tumors are unresectable either due to underlying liver cirrhosis or advanced oncologic stage of the disease; therefore, HCC continues to have a poor prognosis. The underlying cirrhosis in the patients of HCC precludes surgical resection. In such patients, LT is a potentially curative treatmentand is the best treatment option for patients with decompensated cirrhosis as LT surgery removes the tumor along with the underling cirrhosis and cures the portal hypertension. LT as a treatment for early-stage HCC in patients within Milan's criteria is well-established in the
United States and Europe with a 5-year survival rates of $>70 \%$, comparable to those of noncancer liver recipients [2,3]. However, several groups argued that the Milan criteria were too strict and should be expanded to enable patients with slightly more advanced HCC to benefit from LT.

Yao et al. [4] proposed expanding selection criteria known as the University of California at San Francisco (UCSF) criteria. Using these criteria, a 5-year survival rate of $75.2 \%$ after LT was achieved. As the cohort of HCC patient population continues to increase, many institutional studies have analyzed the implications of expanding the criteria for HCC patients that are beyond Milan's criteria. In effort to expand the criteria, "up-to seven" criteria were introduced 
by original Milan group which achieved a 5-year overall survival rate of $71.2 \%$ [5]. Lei et al. [6], in their retrospective analysis of 90 patients who underwent LT using up-to-seven criteria, 76.6\% patients could achieve 5-year survival [6].

We use our institute's criteria for LT in patients with HCC which includes all HCC patients without extra-hepatic metastasis and macrovascular tumor thrombosis irrespective of tumor size [1]. An overall 47\% 5-year survival can be achieved in HCC patients beyond UCSF criteria and the survival further improves in absence of micro and macrovascular tumor thrombosis on explant liver pathology. Advanced HCC patients with solitary adrenal metastasis are one of the subsets of advanced HCC patients that can be benefitted by LT if the solitary metastasis can be resected. The incidence of adrenal metastasis in HCC patients is reportedly $8.4 \%$ in autopsy studies and $1-3.9 \%$ in clinical studies $[7,8]$. Some authors [9] advocate resection of isolated metastatic lesions. However, frequent association with advanced liver disease and extra adrenal metastasis deters patients from surgical interventions.

Locoregional therapies and resection of the metastatic lesion along with hepatectomy for the primary HCC remain curative modalities of treatment. However, in presence of advanced liver cirrhosis LT is the only feasible treatment option. In such patients of HCC with solitary metastasis to the adrenal gland without any other extra-hepatic disease can still be managed by local resection of the adrenal gland metastasis and sequential LT. There are no proven reports regarding the guidelines of the management of such patients. If metastasis can be resected without evidence of any other systemic spread, such patients can still be benefitted by sequential LT. Herein we present our experience of sequential 4 cases of HCC solitary metastasis to adrenal gland that were treated by local resection followed by LT.

\section{Patients and Methods}

Database of 937 patients that underwent LT from September 2002 to December 2017 at china medical university hospital was retrospectively analyzed. The registry of 402 HCC patients that underwent LT was searched and HCC patients with solitary adrenal metastasis were evaluated for the outcome after LDLT. Four such patients with a minimum follow up of 24 months after LDLT were identified and retrospectively analyzed. All the four patients in this study had unresectable HCC due to underlying cirrhosis with solitary adrenal metastasis without any other extrahepatic spread. HCC was diagnosed by typical appearance on computed tomography (CT) imaging that was confirmed by postoperative histopathological analysis. The adrenal gland metastasis was confirmed by pre-LT for 2 years. scan in all these patients.

The retrospective data of past medical conditions and demographics were collected. From the point of study enrollment of the patients, the retrospective as well as the prospective data such as laboratory parameters, liver function profile and imaging studies were collected at regular intervals till the last follow up as per the institute protocol described elsewhere [9]. One patient (Case 2) in this series underwent left adrenal gland excision bearing the solitary metastasis followed by LDLT after six months of initial surgery. For the remaining three patients, adrenalectomy, and LDLT were done as a single stage procedure.

The technique of adrenal gland excision is beyond the scope of this article, hence described briefly. For left adrenalectomy, the lesser sac was entered by reflecting the omentum from the transverse colon. Left lower margin of the pancreas was dissected to gain access to the left adrenal gland. After the tumor location in adrenal gland was confirmed by manual palpation and intraoperative ultrasound examination, the left adrenal gland was excised by dividing the inflow and outflow. In patients with metastasis to right adrenal gland, the excision of the gland was done during recipient hepatectomy after mobilizing the right lobe and carefully dissecting the adrenal gland off the segment six.

\section{Postoperative follow up and Immunosuppression}

The immunosuppression in post-transplantation were as per the institution guidelines [10]. From February 2012, we routinely add everolimus as primary immunosppressant along with tacrolimus with a dose of $0.5 \mathrm{mg}$ twice a day with a target trough levels $3-5 \mathrm{ng} / \mathrm{ml}$. All the patient underwent postoperative doppler ultrasound examination to evaluate the inflow and outflow of the liver graft. CT imaging of the liver was done at three months posttransplantation followed by every six months thereafter for 2 years. Alfa fetoprotein (AFP) levels were monitored in all the patient. Any rise in AFP was considered as prognostically significant.

\section{Result}

Four patients (mean age, 53 years; M: F, 2:2) underwent LDLT for HCC with single adrenal metastasis (right adrenal gland=2 patients; and left adrenal gland $=2$ patients). Figure 1 shows the PET scan images showing the adrenal gland metastasis.

\section{Case 1}

This 50-year male patient of liver cirrhosis with a history of encephalopathy and variceal bleeding was diagnosed to have multiple HCCs over S5-7 and suspected right portal vein thrombosis. PET scan revealed right adrenal gland metastasis with no other extrahepatic metastatic disease (Figure 1A). Liver resection for HCC was not feasible due to Child C functional status of patient. LDLT with right adrenalectomy was performed. At $33^{\text {rd }}$ post-LDLT month patient developed multiple lung metastases and received chemotherapy. Patient expired at $52^{\text {nd }}$ month post LDLT due to intra-abdominal infection and overwhelming sepsis.

\section{Case 2}

This 59-year-old female was diagnosed to have hepatitis $C$ virus related HCC. During routine workup of the patient she was found to have metachronous left adrenal gland solitary metastatic lesion (Figure 1B). Due to underlying cirrhosis, hepatectomy was not possible. Hence, left adrenalectomy was performed, and patient received TACE for HCC. Sequential LDLT was performed after 7 months of adrenal gland resection as her liver functions deteriorated after second TACE that she received. After a follow up of 59 months, patient still remains recurrence free with normal graft functions. 


\section{Case 3}

43-year-female with a chronic hepatitis B viral infection status was diagnosed to have multiple HCC with left adrenal gland metastasis (Figure 1C). She received ABOi LDLT and left adrenalectomy as a single stage surgery. On second postoperative day, patient developed hepatic artery thrombosis that was treated by re-exploration and re-do hepatic arterial anastomosis. Her further course was without any complications and was discharged in third week post LDLT with stable liver functions. Patient developed lung metastasis and died due to respiratory failure after 28 months post-LDLT.

\section{Case 4}

This 61-year-old male with chronic HBV carrier status was diagnosed to have HCC with solitary extrahepatic metastasis at right adrenal gland (Figure 1D). He received LDLT with left adrenalectomy as a single stage surgery. However, patient developed intrahepatic recurrence at 9 months post-LDLT and died due to liver graft failure (Table 1). In fourth patient, the histopathology showed mixed variant HCC with cholangiocarcinoma examination of the explant liver. The AFP levels were less than 100ng/ml. Follow up period ranged from 9 months to 59 months. Maximum survival achieved was 59 months in second patient who continues to have recurrence free survival.
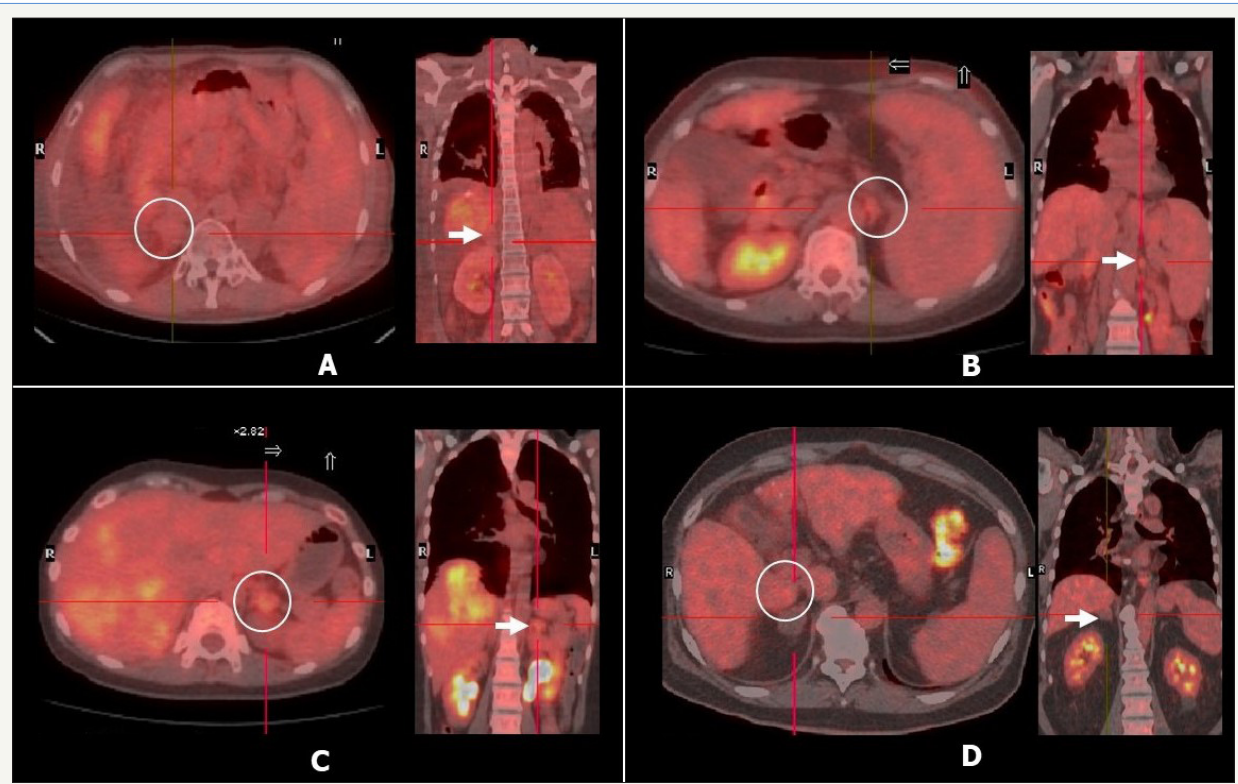

Figure 1: PET-CT images of the patients showing solitary adrenal gland metastasis. A,B, C and D images show the adrenal gland metastasis in patient 1 , patient 2 , patient 3 and patient 4 in this series, respectively.

Table 1: Characteristics of patients.

\begin{tabular}{|l|c|c|c|c|c|c|c|c|c|c|c|}
\hline & \multicolumn{2}{|c|}{ Recipient } & AFP at LT(ng/ml) & \multicolumn{2}{|c|}{$\begin{array}{c}\text { Pre LT Dx of Adrenal } \\
\text { Gland }\end{array}$} & $\begin{array}{c}\text { Pre/Post LT of TNM } \\
\text { Stage }\end{array}$ & \multicolumn{4}{|c|}{ Outcome } \\
\hline & Age & Sex & & CT & PET & T & N & M & $\begin{array}{c}\text { R/L } \\
\text { side }\end{array}$ & OS (months) & Alive/Death \\
\hline Patient 1 & 50 & M & 25 & $(+)$ & $(+)$ & $3 a / 4$ & $0 / 0$ & 1 & R & 52 & $\begin{array}{c}\text { Died (Lung metas- } \\
\text { tasis) }\end{array}$ \\
\hline Patient 2 & 60 & F & 13.18 & $(+)$ & $(+)$ & $2 / 2$ & $0 / 0$ & 1 & L & 59 & Alive \\
\hline Patient 3 & 43 & F & 43.38 & $(+)$ & $(+)$ & $3 a / 3 a$ & $0 / 0$ & 1 & L & 28 & $\begin{array}{c}\text { Died (Lung metas- } \\
\text { tasis) }\end{array}$ \\
\hline Patient 4 & 61 & M & 90.05 & $(+)$ & $(+)$ & $2 / 2$ & $0 / 0$ & 1 & R & 9 & $\begin{array}{c}\text { Died (Lung metas- } \\
\text { tasis) }\end{array}$ \\
\hline
\end{tabular}

*patient 4 in this series had mixed HCC-Cholangiocarcinoma variant on histopathology examination.

\section{Discussion}

Metachronous adrenal gland metastasis in patients with HCC is not uncommon with incidence ranging from 1-2.4\% [11]. The worldwide experience of the surgical treatment in such situation remains limited [12]. Previous studies showed that the patients with isolated extrahepatic metastasis and preserved liver functions may generally benefit from a surgical resection and/or aggressive local therapies that are advocated for both primary tumor and metastatic lesions $[13,14]$. However, if the HCC is unresectable due to underlying cirrhosis, the condition becomes potentially untreatable. Although few reports describe post-LT recurrence of HCC in the adrenal gland with successful curative adrenal metastasis resection $[15,16]$, the role of LT for unresectable HCC with metachronous solitary adrenal metastasis has been never 
described before. This case series is the first report of modest longterm outcome after LDLT for HCC along with adrenal metastasis resection either as a single stage surgery or sequential surgery.

Neoplastic recurrence or extrahepatic metastasis in HCC occurs in lungs, adrenal gland or bones with a reported median survival of 4.9 months [17]. The patients of HCC that have multiple metastases could only be offered supportive form of therapy that includes palliative chemotherapy. However, previous studies showed that a solitary metastasismighthavetheopportunity of surgical resection. Lyonetal. (13) reported a mean survival of 38 months in 7 patients who underwent adrenalectomy for metastatic HCC. Two patients in their series died in the postoperative period due to pulmonary embolism $(n=1)$ and acute pancreatitis $(n=1)$. No recurrence of primary disease was reported. The outcome after surgical resection of the isolated adrenal gland metastasis after LT has been reported to have a 24-months disease free survival [18]. In presence of unresectable HCC due to underlying cirrhosis, LT remains the only option if the adrenal metastasis can be resected. Our present series describes adrenal gland $r$ metastasis resection prior to LT and/or along with LT as a single stage procedure. Except for one patient (patient 4 in this series) all the patients had more than 24-months recurrence free survival and longest survival achieved is 59 months.

Alternatively, a variation of nonsurgical treatment options like percutaneous ethanol injection, TACE, radiation, and radiofrequency ablation were also reported, and recent chemotherapeutic protocols achieved palliative control of adrenal metastasis with a median survival of 11 months. The survival, however, very less as compared to the adrenalectomy after curative treatment for primary HCC which improves survival rates [19-21].

Furthermore, depending on the curative HCC treatment, recipients of LT had a longer recurrence-free and overall survival rates compared to patients that received curative liver resection for HCC [17]. As experience about role of LT in unresectable HCC with adrenal gland metastasis remains less, the present series is first reported experience in this regard that describes successful outcome after LT with a single stage adrenalectomy for HCC metastasis. The fourth patient in this series had early recurrence in lungs that developed within a year after LT and curative resection. It could be secondary to metachronous lung lesions which were initially absent on PET scan. The AFP levels were monitored that showed increased levels to $90 \mathrm{ng} / \mathrm{ml}$ from initial normal levels. Moreover, the histopathological analysis showed HCC with cholangiocarcinoma which has more propensity for extrahepatic metastasis.

Three patients developed extrahepatic recurrence of HCC in the lungs without any evidence of hepatic recurrence or other extrahepatic metastasis. Palliative chemotherapy could be delivered to such patients with recurrent HCC after LT with tolerable toxicity that may improve survival of the patients. Capecitabine plus oxaliplatin chemotherapeutic regimen shows a modest anti-tumor activity with tolerable toxicities in patients with advanced HCC and can be administered in patients with poorly differentiated HCC with recurrence at extrahepatic locations. A minimum of 2-years recurrence free survival in three patients in this series highlights earlier reported beneficial elements, such as complete surgical removal of the underlying disease, unusual sensitivity to adjuvant therapy, endocrine influence and other complex factors. Therefore, the aggressive treatment for unresectable HCC with underlying cirrhosis and extrahepatic solitary metastasis in the adrenal gland by LT and adrenalectomy as a single stage procedure can improve long term survival despite the risk for recurrence and perioperative challenges after prior major abdominal surgery.

Present study opens a new frontier in managing patients of advanced HCC with a modest long-term survival with recurrence free survival of 59 months in one patient. However, the small number of patients and the retrospective design are the major limitations of this study. The similar data published in past that described long term outcome after adrenalectomy after LT for HCC recurrence in adrenal gland highlights the feasibility and safety of such procedure even when combined with LT as single stage surgery.

\section{References}

1. Jeng LB, ThoratA, Yang HR (2016) Surgical Management of Hepatocellular carcinoma: Current Asian perspectives and controversies- How far we have come? Med Sci Rev 3: 73-84.

2. Mazzaferro V, Regalia E, Doci R, Andreola S, Pulvirenti A, et al. (1996) Liver transplantation for the treatment of small hepatocellular carcinomas in patients with cirrhosis. N Engl J Med 334(11): 693-699.

3. Chen DS (2010) Toward elimination and eradication of hepatitis B. J Gastroenterol Hepatol 25(1): 19-25.

4. Yao FY, Ferrell L, Bass NM, Watson JJ, Bacchetti P, et al. (2001) LT for hepatocellular carcinoma: Expansion of the tumor size limits does not adversely impact survival. Hepatology 33(6): 1394-1403.

5. Mazzaferro V, Llovet JM, Miceli R, Bhoori S, Schiavo M, et al. (2009) Predicting survival after liver transplantation in patients with hepatocellular carcinoma beyond the Milan criteria: a retrospective, exploratory analysis. Lancet Oncol 10(1): 35-43.

6. Lei JY, Wang WT, Yan LN (2013) Up-to-seven criteria for hepatocellular carcinoma liver transplantation: A single center analysis. World J Gastroenterol 19: 6077-6083.

7. Natsuizaka M, Omura T, Akaike T, Kuwata Y, Yamazaki K, et al. (2005) Clinical features of hepatocellular carcinoma with extrahepatic metastases. J Gastroenterol Hepatol 20(11): 1781-1787.

8. Yamakado K, Anai H, Takaki H, Sakaguchi H, Tanaka T, et al. (2009) Adrenal metastasis from hepatocellular carcinoma: radiofrequency ablation combined with adrenal arterial chemoembolization in six patients. Am J Roentgenol 192(6): W300-W305.

9. Park JS, Yoon DS, Kim KS, Choi JS, Lee WJ, et al. (2007) What is the best treatment modality for adrenal metastasis from hepatocellular carcinoma? J Surg Oncol 96(1): 32-36.

10. Thorat A, Jeng LB, Yang HR, Yeh CC, Hsu SC, et al. (2017) Assessing the role of everolimus in reducing the HCC recurrence after living donor liver transplantation for patients within UCSF criteria: Re-inventing the role of mammalian target of rapamycine inhibitors. Ann Hepatobiliary Pancreat Surg 21(4): 205-211.

11. Jeng LB, Thorat A, Hsieh YW, Yang HR, Yeh CC, et al. (2014) Experience of using everolimus in the early stage of living donor liver transplantation. Transpl Proc 46(3): 744-748.

12. Chen SW, Wang S, Wang B, Li WD, Yan S, et al. (2011) Metachronous pulmonary and adrenal metastases after liver transplantation for hepatocarcinoma. World J Surg Oncol 9(1): 156. 
13. Jung J, Yoon SM, Park HC, Nam TK, Seong J, et al. (2016) Radiotherapy for Adrenal Metastasis from Hepatocellular Carcinoma: A Multi-Institutional Retrospective Study (KROG 13-05). PLoS One 11(3): e0152642.

14. Pandey D, Tan KC (2008) Surgical resection of adrenal metastasis from primary liver tumors: a report of two cases. Hepatobiliary Pancreat Dis Int 7(4): 440-442.

15. Rubio E, Gonzalez J, Jimenez M, Lucena JL, Gimenez L, et al. (2009) Right adrenal metastases of hepatocarcinoma after liver transplantation: case report and literature review. Transplant Proc 41(3): 1067-1069.

16. Ha TY, Hwang S, Ahn CS, Kim KH, Lee YJ, et al. (2014) Resection of metachronous adrenal metastasis after liver resection and transplantation for hepatocellular carcinoma. Dig Surg 31(6): 428-435.

17. Uka K, Aikata H, Takaki S, Shirakawa H, Jeong SC, et al. (2007) Clinical features and prognosis of patients with extrahepatic metastases from hepatocellular carcinoma. World J Gastroenterol 13(3): 414-420.
18. Berger Y, Spivack JH, Heskel M, Aycart SN, Labow DM, et al. (2016) Extrahepatic metastasectomy for hepatocellular carcinoma: Predictors of long-term survival. J Surg Oncol 114(4): 469-474.

19. Hasegawa T, Yamakado K, Nakatsuka A, Uraki J, Yamanaka T, et al. (2015) Unresectable adrenal metastases: Clinical outcomes of radiofrequency ablation. Radiology 277(2): 584-593.

20. Berretta M, Lleshi A, Di Benedetto F, Bearz A, Spina M, et al. (2006) Oxaliplatin and capecitabine (Xelox) in association with highly active antiretroviral therapy in advanced hepatocarcinoma HIV/HCV-infected patients. Ann Oncol 17(7): 1176-1177.

21. Shimoda M, Ghobrial RM, Carmody IC, Anselmo DM, Farmer DG, et al. (2004) Predictors of survival after liver transplantation for hepatocellular carcinoma associated with Hepatitis C. Liver Transpl 10(12): 1478-1486.

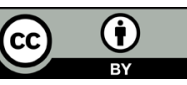

Creative Commons Attribution 4.0 International License

For possible submissions Click Here

\section{Submit Article}

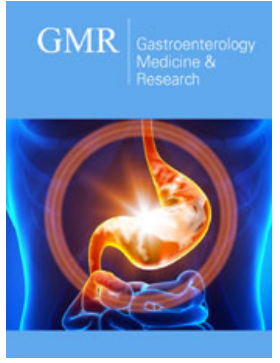

Gastroenterology Medicine \& Research

\section{Benefits of Publishing with us}

- High-level peer review and editorial services

- Freely accessible online immediately upon publication

- Authors retain the copyright to their work

- Licensing it under a Creative Commons license

- Visibility through different online platforms 